\title{
Aorto-bi-Femoral Bypass for Juxtarenal Aortic Occlusion in a Patient with Multiple Co- Morbidities: Report of a Challenging Case
}

\author{
AHM Bashar ${ }^{1}$, S Samad ${ }^{1}$, ASMI Hossain ${ }^{2}$, MN Sabah ${ }^{1}$, K Hasan $^{2}$, M Alauddin $^{3}$ \\ ${ }^{1}$ Department of Vascular Surgery, NICVD, ${ }^{2}$ Department of Cardiac Surgery, NICVD, \\ ${ }^{3}$ Department of Anaesthesia, NICVD
}

\begin{abstract}
Key Words : Aorto-bi-femoral bypass, Juxtarenal aortic occlusion, Ischemic heart disease, malnutrition

Aorto-bi-femoral bypass for aorto-iliac occlusive disease is a fairly common procedure in vascular surgical practice. The procedure, despite its extensive nature, is generally well tolerated particularly by those having an infrarenal type of aortic occlusion. Patients having a juxtarenal aortic occlusion require a considerably more expeditious surgery necessitating maneuvers to protect the renal arteries. Surgical risk increases significantly in those having multiple co-morbid conditions. We report here a successful case of aorto-bi-femoral bypass for juxtarenal aortic occlusion in a patient having multiple co-morbid conditions like ischemic heart disease, occluded right renal artery with chronic kidney disease, severe malnutrition, hypertension and chronic obstructive pulmonary disease.
\end{abstract}

(Cardiovasc. j. 2014; 6(2): 170-174)

\section{Case Report:}

A 60-year-old male presented with a large dry gangrene over the antero-lateral aspect of the middle third of the thigh with severe rest pain for which he had been suffering for 6 months. He gave a history of intermittent claudication with buttock claudication for the last 3 years. The patient was non-diabetic but hypertensive for the last 10 years for which he took irregular medication. He didn't give any history of chest pain but reported having cough and breathlessness. He has been a heavy smoker consuming about 20 sticks of cigarette per day for the last 40 years. His family history is positive for hypertension and ischemic heart disease. Drug history included irregular antihypertensive medication, analgesics mostly of NSAID group and antiulcerants.

On physical examination, the patient appeared ill-looking and grossly emaciated with a body weight of $36 \mathrm{~kg}$. He was slightly anemic but nonicteirc. Blood pressure was 190/100 mm Hg. Peripheral pulses were absent in both lower limbs. The lower limbs were cold and grossly wasted with intact sensory and motor functions.
The dry ulcer in the left thigh measured about $7.0 \mathrm{X} 5.0 \mathrm{~cm}$ in dimension, and was found covered by a thick crust. Examination of the cardiovascular and neurological systems revealed no significant abnormality. On examination of the respiratory system, the breath sound was vesicular with prolonged expiration. A few ronchi could be heard over the middle and lower zone of both lungs.

Chest X-ray showed emphysematous change in both lungs. Electrocardiogram was within normal limits. Echocardiogram showed mild anterior wall hypokinesis with a left ventricular ejection fraction of $53 \%$. His laboratory data revealed mild anemia with a hemoglobin level of $10.5 \mathrm{gm} / \mathrm{dl}$, mild renal impairment with a serum creatinine level of $1.5 \mathrm{mg} / \mathrm{dl}$. Urine examination did not show significant proteinuria. Duplex vascular ultrasound established a diagnosis of aorto-iliac occlusive disease (AIOD) having good distal run-off in both lower limbs with visualization of the below-knee arteries.

The patient underwent peripheral and coronary angiogram through the right brachial route ensuring adequate hydration in the immediate

Address of Correspondence : Dr. Abul Hasan Muhammad Bashar, Department of Vascular Surgery, National Institute of Cardiovascular Diseases, Dhaka, Bangladesh.E-mail: ahmbashar@yahoo.com 
preprocedural period. Peripheral angiogram showed total occlusion of the abdominal aorta just below the renal arteries with ostial occlusion of the right renal and inferior mesenteric arteries (Figure 1). Femoral artery was visualized on both sides through collaterals. Below-knee arteries, however, were not visualized. Coronary angiogram revealed a double-vessel coronary artery disease (CAD) with chronic total occlusion of the proximal right coronary artery and 60$70 \%$ stenosis of the distal left circumflex artery (Figure 2).

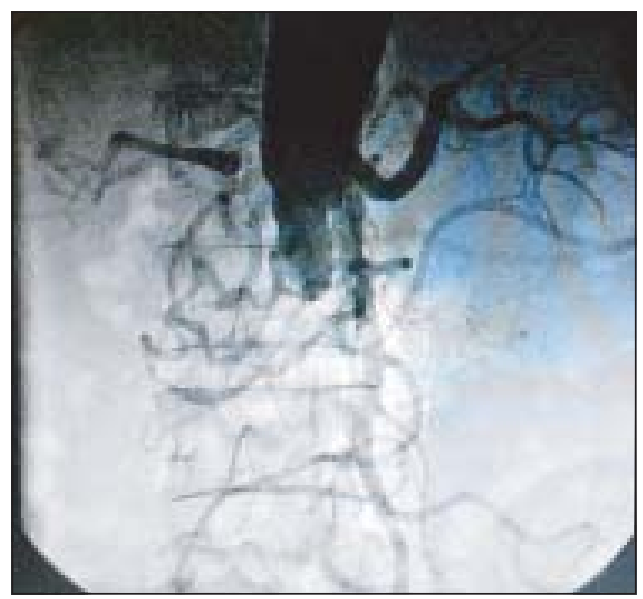

Fig.-1: Abdominal aortogram showing total occlusion of the abdominal aorta just below the origin of the renal arteries. Ostial occlusion of the right renal artery is also seen.

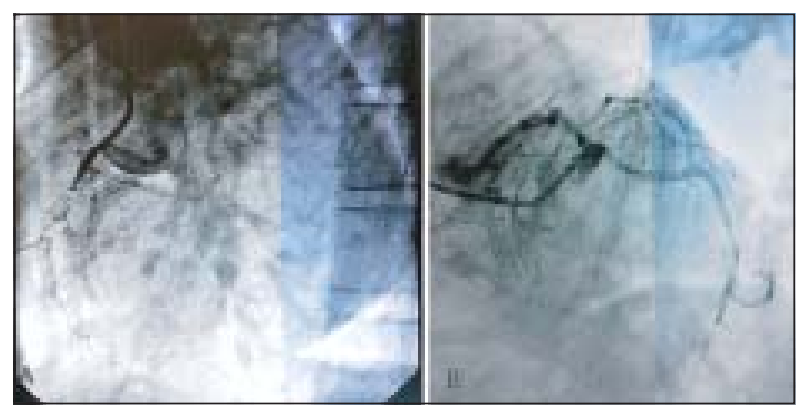

Fig.-2: Coronary angiogram. A. chronic total occlusion of the right coronary artery. B. 60-70\% stenosis of the distal left circumflex artery

Based on the angiographic findings, it was judged that the patient required an aorto-bifemoral bypass (ABFB) with Y-graft. The timing of surgery, however, was difficult to decide given the multiple comorbidities of the patient. The decision to do it early was prompted by symptoms of the patient which were severe ischemic rest pain and ongoing tissue loss. The patient party was counseled regarding the risks of the procedure. A cardiologist was involved who recommended peripheral revascularization with moderately increased cardiac risk. Opinion from a nephrologist was also sought who advised early peripheral revascularization considering the ongoing tissue loss in the lower limb whichhe opined- might further aggravate renal function. The party gave a written consent with the understanding of the added risks of surgery and an ABFB was panned in a specialized hospital.

Under epidural anesthesia, the common, superficial femoral and deep femoral arteries were exposed through longitudinal incisions in both groins. Then the abdominal aorta was approached transperitoneally through a midline incision. Adequate mobilization of the gut loops was achieved by dissection at the duodenojejunal flexure. First, control of the left renal vein was taken. Then the suprarenal abdominal aorta and the renal arteries were controlled. Systemic heparinization was done and the abdominal aorta was completely transected at the occluded segment about $2.5 \mathrm{~cm}$ below the level of the renal arteries. The aorta was found totally occluded by atheromatous debris with thrombotic material at the core. Thromboendarterectomy was then performed from below under careful digital control. During thromboendarterectomy, small bulldog clamps were placed on the renal arteries to prevent embolization of debris.

Once the proximal aorta was cleared of atheromatous debris, it was flushed with heparinized saline and the aortic clamp was placed below the renal arteries. Distal aortic stump was closed with mattress sutures. ABFB was then fashioned using a14X7 mm polytetrafluoroethylene (PTFE) Y-graft prosthesis (Atrium Medical Corporation, 5 Wentworth Drive, Hudson, New Hampshire, USA). Proximal anastomosis was performed in an end-to-end fashion using 3-0 polypropylene suture which was followed by distal anastomoses using 6-0 polypropylene suture. Proper 
hemostasis was obtained. Posterior peritoneum was re-approximated and the abdomen closed in layers leaving a drain. The groin wounds were also closed after proper hemostasis without drain.

The immediate postoperative period of the patient was complicated by an episode of intractable vomiting attributable to paralytic ileus which was managed conservatively. There was a transient rise in the serum creatinine level to $2.8 \mathrm{mg} / \mathrm{dl}$ with decreased output. This was managed by careful volume and electrolyte correction in close consultation with a nephrologist. The patient, otherwise, recovered well to be discharged on the $10^{\text {th }}$ postoperative day. All the surgical wounds healed well except the left groin wound where lymphorrhea occurred, necessitating long term wound care. The patient was relieved of his ischemic pain immediately after the operation, pedal pulses became palpable and the patient has been mobile ever since. Postoperative Duplex scan revealed satisfactory flow in both lower limb arteries including the pedal arteries (Figure 3,4).

About 4 weeks after the operation, the patient developed progressive abdominal distension and bilateral leg edema. Abdominal ultrasound revealed e ascitis and bilateral renal parenchymal disease without evidence of chronic liver disease. Laboratory data revealed hypoproteinemia (serum total protein $4.6 \mathrm{gm} / \mathrm{dl}$, albumin $2.2 \mathrm{gm} /$ dl) with altered albumin/globulin ratio. Liver function was otherwise normal. Asitic fluid study showed a transudative nature of the fluid without evidence of hemorrhage or infection. Correction of albumin was done and ascitis and leg edema gradually subsided. The patient was referred to plastic surgical care for his thigh ulcer.

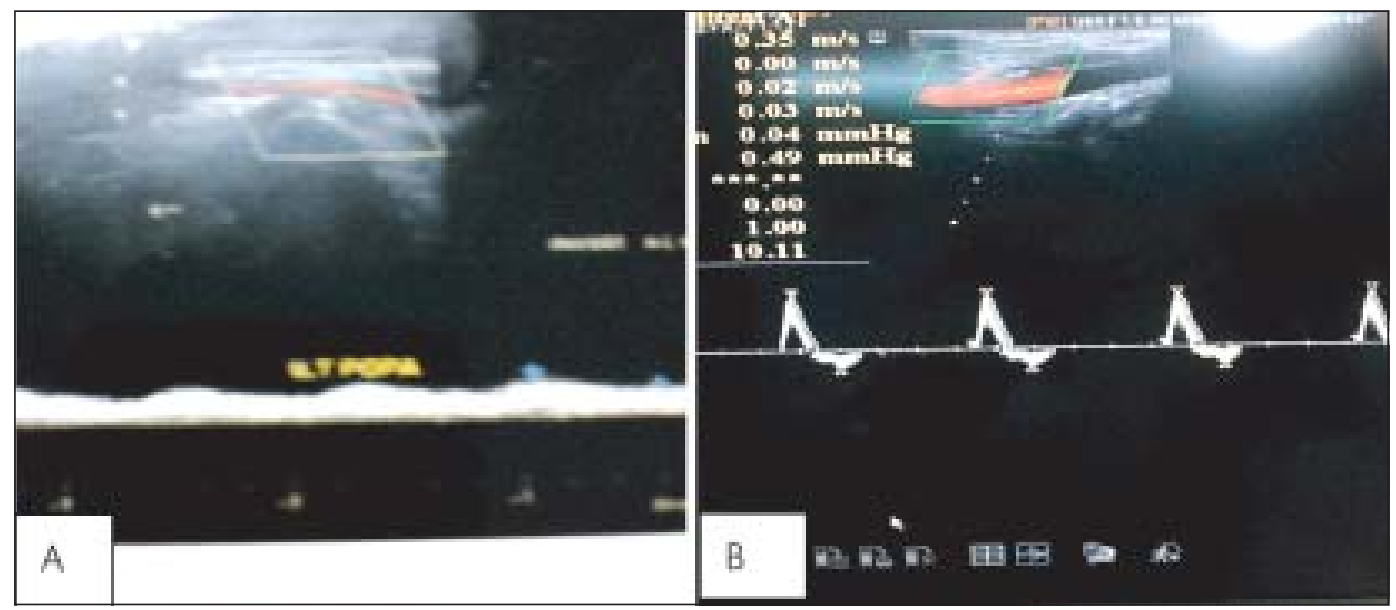

Fig.-3: Pulsed wave Doppler spectral pattern and velocity in the left Popliteal artery. A. Low velocity monophasic flow before surgery B. Normal triphasic flow with satisfactory velocity after surgery.

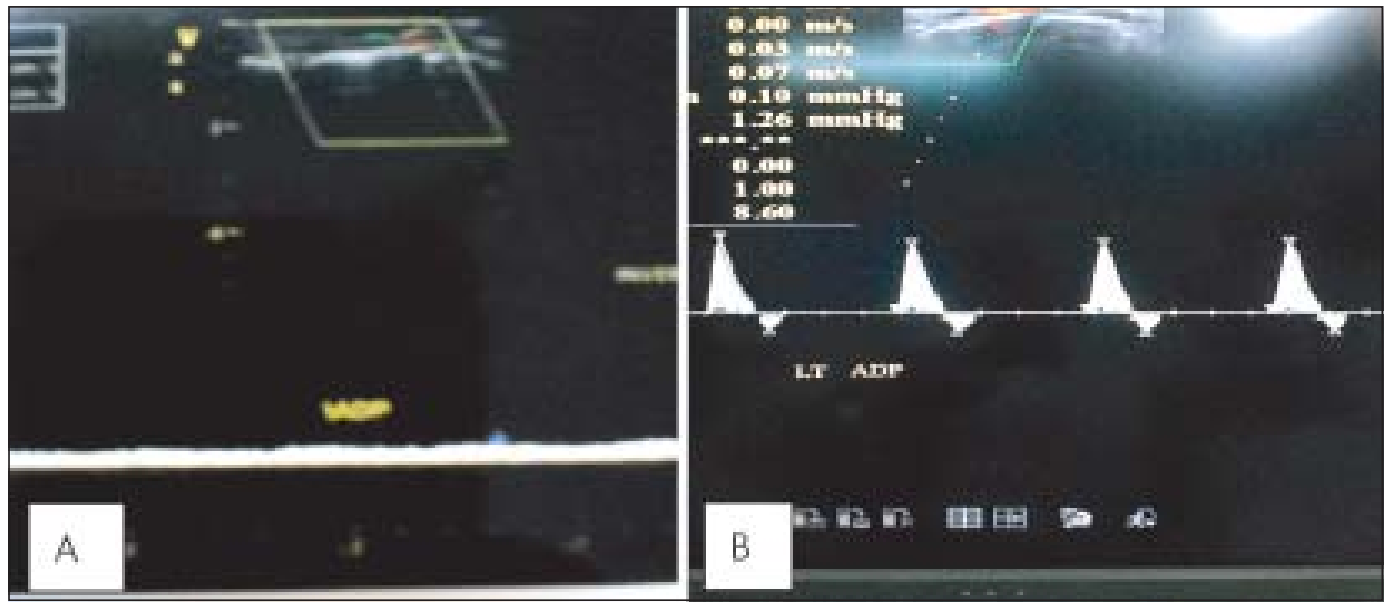

Fig.-4: Pulsed wave Doppler spectral pattern and velocity in the left Arteria Dorsalis Pedis. A. Low velocity monophasic flow before surgery B. Normal triphasic flow with satisfactory velocity after surgery. 


\section{Discussion:}

ABFB surgery is basically performed for two types of disease conditions- aorto-iliac occlusions and aorto-iliac aneurysms. In the vascular surgical practice of Bangladesh, the former far outnumbers the latter. This is supported by the disease profile encountered at the National Institute of Cardiovascular Diseases (NICVD), Dhaka. ${ }^{1}$ In the western countries and in far-east, the trend, however, is different and application of this procedure is more for aneurysmal diseases. ${ }^{2}$ This raises a potentially important issue- is there a geographical or racial variation in the pattern of these diseases or is it just that a large proportion of the aneurysmal diseases remains undiagnosed in our country?

Mortality for ABFB is 2-5\% in experienced hands with optimum set-up; it may be higher in low volume centers. ${ }^{3,4}$ In our country, NICVD is the only center where the procedure is routinely performed. In-hospital mortality has been low at $4.6 \% .^{5}$ This series published in 2011 , however, included patients who didn't have significant $\mathrm{CAD}$ or chronic kidney disease (CKD). The only CAD patient who underwent surgery about 2 weeks after coronary stenting died in the immediate post-operative period. ${ }^{5}$ The present case had double-vessel CAD with echocardiographic evidence of anterior wall hypokinesis. Surgery after correction of the coronary lesion could have been a strategy. The current recommendations from the American Heart Association regarding major non-cardiac surgery after coronary stenting is that surgery should be deferred by at least 30 days in case of bare metal stent and by a year when drug eluting stent is used. ${ }^{6}$ Adherence to this guideline in the present case would mean considerable delay in surgery which would inevitably lead to progressive tissue loss in the lower limb and possible limb loss.

The most common causes of death after ABFB surgery are perioperative myocardial infarction and stroke. ${ }^{7}$ Other causes of death include renal and respiratory failure. Having documented CAD, CKD and COPD, our patient was at high risk for adverse cardio-respiratory and renal events. For renal protection, we ensured adequate perioperative hydration and took measures to prevent embolization of debris in to the renal arteries during thromboendarterectomy on the aorta.

Operation in the present case was performed under epidural anesthesia. Advantages of this modality of anesthesia over general anesthesia with endotracheal intubation for patients with compromised lung function has been well described. ${ }^{7}$ As additional protective measures for respiratory failure, we ensured cessation of smoking about a month prior to surgery and subjected the patient to chest physiotherapy. Rigorous hemodynamic monitoring with an aim to prevent sudden hypotension was also instituted during the operation and also in the immediate post-operative period. Inotropes such as dopamine and noradrenaline were used with meticulous dosage titration. These, we believe, contributed to the positive outcome in this patient. The patient also had severe malnutrition with a body weight of only $36 \mathrm{~kg}$ before operation which was the consequence of severe anorexia that the patient had for a period over 6 months in the preoperative period. This could have been due to the chronic inflammatory process in the body as well as the severe ischemic rest pain. No specific measures were taken to correct the nutritional status before the operation. We believe that correction of hypoproteinemia in consultation with a nutritionist could reduce the risk of hypoproteinemic edema that the patient had in the postoperative period.

In conclusion, juxtarenal aortic occlusion with multiple comorbidities like CAD, CKD, COPD and severe malnutrition constitutes a high-risk disease condition. Benefit of surgery in such patients must be carefully weighed against the risks before making a decision. When surgery is decided, a team of experts with representation from appropriate specialities should be involved in the perioperative care of these patients.

\section{References:}

1. Unpublished data from the department of vascular surgery, National Institute of Cardiovascular Diseases (NICVD), Dhaka, Bangladesh.

2. Steckmeier B. Epidemiology of aortic disease: aneurysm, dissection, occlusion. Radiologe 2001; 41(8):624-632. 
3. Dimick JB, Cowan JA Jr, Henke PK, et al. Hospital volume-related differences in aorto-bifemoral bypass operative mortality in the United States. J Vasc Surg 2003; 37(5):970-975.

4. Szilagyi DE, Elliot JP, Jr Smith RF, Reddy DJ, McPharlin M. A 30-year survey of the reconstructive surgical treatment for aortoiliac occlusive disease. J Vasc Surg 1984; 3:421-435.

5. Bashar AHM, Hossain GMM, Hakim ME, et al. Aorto-biFemoral bypass for aorto-iliac occlusive disease: Recent Experience at the National Institute of Cardiovascular Diseases (NICVD). Cardiovasc J 2011; 4(1):26-31.
6. Fleisher LA, Beckman JA, Brown KA, et al. ACC/AHA 2007 guidelines on perioperative cardiovascular evaluation and care for noncardiac surgery: a report of the American College of Cardiology/American Heart Association Task Force on Practice Guidelines (Writing Committee to Revise the 2002 Guidelines on Perioperative Cardiovascular Evaluation for Noncardiac Surgery). J Am Coll Cardiol 2007;50:e159-e241.

7. Menard MT and Belkin M. Aortoiliac Disease: Direct Reconstruction. In Cronenwett JL, Wayne Johnston, eds. Rutherford's Vascular Surgery. Philadelphia, PA: Saunders Elsvier; 2010:1613-32. 